\title{
NaCl Triggers the Sessile-to-Motile Transition of Bacillus subtilis
}

\author{
Prem Anand Murugan ${ }^{1}$, Manish Kumar Gupta ${ }^{2}$, T. Sabari Sankar ${ }^{3}$, Sivasurender Chandran $^{2 *}$
} and Saravanan Matheshwaran ${ }^{1,4,5 *}$

${ }^{1}$ Department of Biological Sciences and Bioengineering, Indian Institute of Technology, Kanpur, India

${ }^{2}$ Department of Physics, Indian Institute of Technology, Kanpur, India

${ }^{3}$ School of Biology, Indian Institute of Science Education and Research, Thiruvananthapuram, India

${ }^{4}$ Centre for Environmental Sciences and Engineering, Indian Institute of Technology, Kanpur, India

${ }^{5}$ Mehta Family Centre for Engineering in Medicine, Indian Institute of Technology, Kanpur, India

Author Contributions: P.A.M., T.S.S, S.C., and S.M. designed research; P.A.M and S.C. performed experiments, All authors analyzed data and contributed to the writing of the paper.

Corresponding authors:

Sivasurender Chandran, schandran@iitk.ac.in

Saravanan Matheshwaran, saran@ iitk.ac.in

Keywords: Biofilm, surface motility, super diffusion, flagella, Bacillus subtilis, $\mathrm{NaCl}$ 


\begin{abstract}
Various chemical cues are known to alter the motile and sessile states of bacteria differentially and, in turn, the formation of biofilms. However, the underlying mechanisms at the cellular and molecular level remain less understood, which severely limits our ability to control biofilms. Here, we systematically studied the effects of $\mathrm{NaCl}$ on the dynamics of biofilm formation across various length scales and the associated changes in the regulation of gene expression in an undomesticated natural isolate of Bacillus subtilis. Interestingly, $\mathrm{NaCl}$ induced significant changes in the architecture of pellicles and yielded systematic increase in lateral expansion rates of biofilms when grown on an agar surface. At the microscopic level, we observed an intriguing $\mathrm{NaCl}$-induced transition from slow and isotropic super diffusion behavior of individual bacterial cells to rapid anisotropic diffusion behavior. The average orientation of the cells (at the edge) along the expanding direction and the increased flagellation in a subpopulation of cells in the presence of $\mathrm{NaCl}$ corroborates well with the observed rapid anisotropic diffusion of cells. Further, the cellular uptake of $\mathrm{NaCl}$ resulted in the downregulation of several genes underlying the formation of biofilms, revealing the role of chemical cues like $\mathrm{NaCl}$ in controlling the gene regulatory circuit underlying the sessile to motile transition. Our study opens a new avenue to decipher the competitive advantage provided to the subcellular populations by $\mathrm{NaCl}$ due to lifestyle switch in Bacillus subtilis.
\end{abstract}




\section{Introduction}

Bacteria are the most abundant and diverse form of life on the planet. In their natural environments, they often live in structurally complex and dynamic multicellular communities called biofilms, where bacteria are embedded in the self-secreted viscoelastic fluids comprised of various macromolecules, including exopolysaccharides (EPS), and proteins that stabilize biofilms (1-3). In contrast to the planktonic state, the multicellular lifestyle provides fitness advantages for the microbes by enhancing their tolerance/resistance to external chemical and mechanical stressors (1-3). Due to the increased tolerance, biofilms often pose major challenges and threats to various sectors, importantly health care (chronic infections, and antimicrobial resistance) and industry (bioremediation, agriculture, food hygiene, and antibiofouling) (1-5). On the other hand, biofilms are essential in facilitating biogeochemical processes, food digestion, immunity, biodegradation, and plant growth (1-4). Thus, given the importance and impact of biofilms, it is imperative to gain a comprehensive understanding of biofilms to limit their perils and expand on their promises.

Numerous studies have demonstrated that bacteria employ highly sophisticated physical, chemical, and biological principles to develop these stable communal forms and to establish intra- and inter-species communication across colonies (1-23). For instance, the development of biofilm invokes a complex interplay of various cellular and biochemical processes (spatiotemporal gene expression changes, gene transfer, and predation) and mechanical processes (cell-cell and cell-substrate interactions, hydrodynamic interactions, and diffusion of nutrients) acting across several length scales (6-23). The growth of bacteria in a self-imposed viscoelastic environment of EPS creates effective in-plane stresses across the entire biofilm, which, in turn, creates three-dimensional architectures $(7,8,10-12,14)$. 
Despite the vast knowledge, there is still a dearth of understanding on several critical aspects of the biofilm lifestyle essential for bacterial survival and transmission. Importantly, the switching between the sessile (biofilms) to motile states, is regulated by complex and diverse mechanisms depending on the environmental signals, effectors, and signal transduction, which are yet to be unveiled (24). The action of chemical-mediated changes in motility mechanisms from motile-to-sessile or vice versa $(1,3,25)$ presumably altering the expression of genes that are involved in motility and biofilm formation is sparsely understood. Since motility can be the key to the success of tackling antibiotic tolerance strongly exhibited by biofilm-forming bacteria, it is imperative to identify the correlations between the chemical cues and the genetic, physiological, and molecular pathways that can activate and mediate motility.

To this end, we investigated the effects of a chemical cue, salt $(\mathrm{NaCl}$, a strong inducer of stress response) on structure formation and dynamics of biofilm in an undomesticated of B. subtilis strain IITKSM1 (26) across length scales. By varying the concentration of $\mathrm{NaCl}$, we observed a systematic variation in the morphology of pellicle and biofilms. While the pellicle forming ability is considerably decreased in the presence of $\mathrm{NaCl}$, we observed a rapid expansion of biofilms on agar surfaces. Concomitantly, at the cellular level, $\mathrm{NaCl}$ induced rapid diffusive movement of bacteria along the direction of lateral expansion, in contrast to the slow superdiffusive behavior in the absence of $\mathrm{NaCl}$. The rapid motility of the cells is supported by the increased flagella biosynthesis upon treatment with $\mathrm{NaCl}$. Thus, our study reveals that $\mathrm{NaCl}$ acts as a switch that triggers the sessile-to-motile transition. Using differential gene expression analysis, we observed the upregulation of motility genes and the downregulation of genes associated with biofilm formation, thereby revealing the molecular mechanisms underlying the observed behavior. To summarize, our study demonstrates how a simple chemical cue can trigger and reprogram gene expression that modulates the dynamics of biofilms. 


\section{Results and discussion}

\section{NaCl-mediated regulation of pellicle formation and biofilm expansion.}

Figure 1 summarizes $\mathrm{NaCl}$-induced changes in the formation of pellicles. A systematic increase in concentration $\left(W_{\mathrm{NaCl}}\right)$ of $\mathrm{NaCl}$ from 0 to 2 wt.\% showed drastic changes in the architecture of pellicles (Fig 1A and SF1A). We quantified these deviations by measuring the number of wrinkles ( $\left.N_{\text {wrinkles }}\right)$ and the area $(A)$ covered by them (Fig 1B). Clearly, $N_{\text {wrinkles }}$ and $A / A_{\text {control }}$ (where, $A_{\text {control }}$ is the area of the wrinkles in the absence of $\mathrm{NaCl}$ ) decreased with an increase in $W_{\mathrm{NaCl}}$. The wrinkled morphology of pellicles has been observed earlier and alluded to the development of in-plane residual stresses due to the confined geometry over which they are growing (27). Using similar arguments, the controlled decrease in $N_{\text {wrinkles }}$ with $W_{\mathrm{NaCl}}$ suggest a systematic decrease in the in-plane stresses developed in the pellicles. Why do the in-plane stresses decrease with $W_{\mathrm{NaCl}}$ ? 

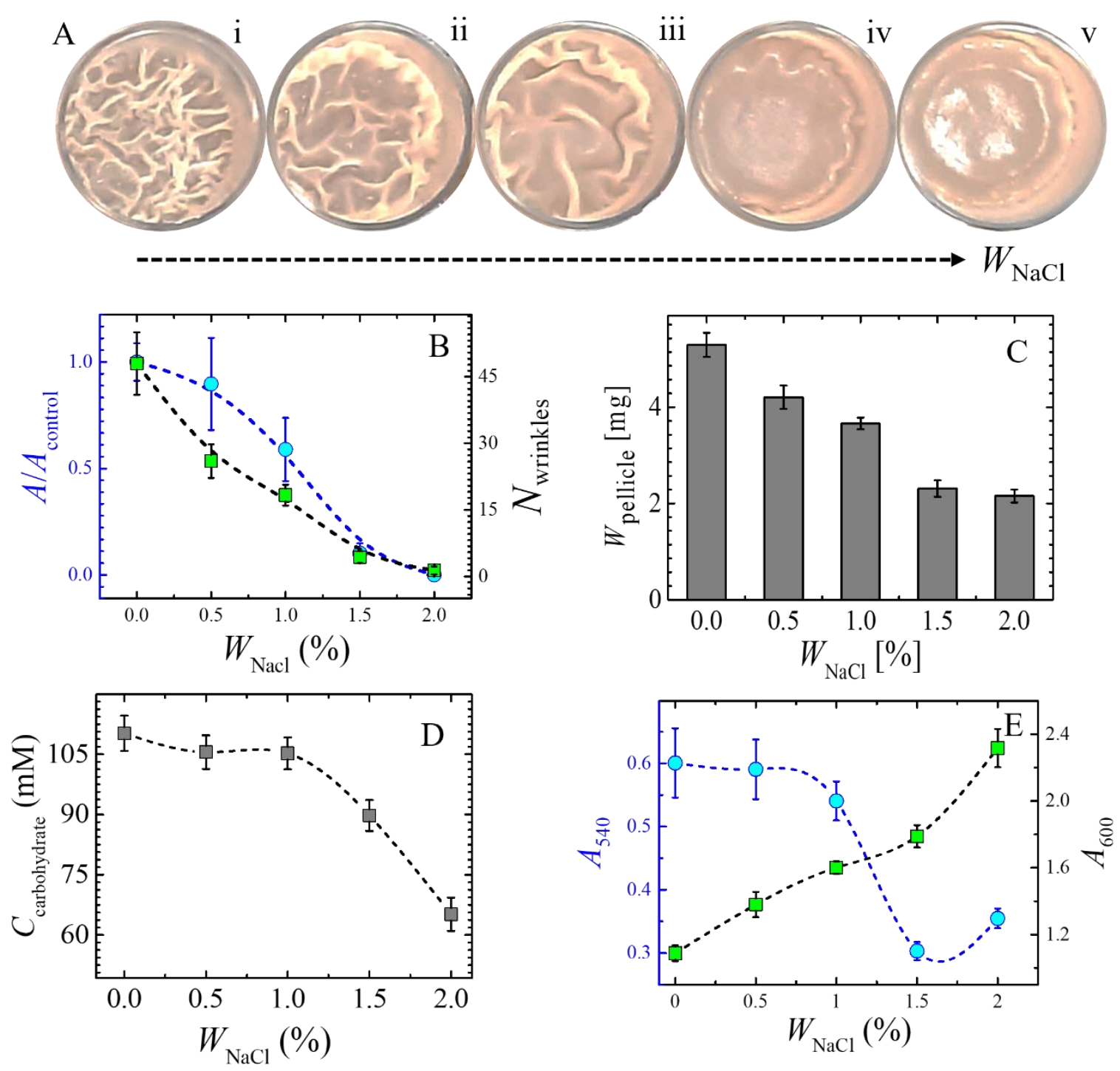

Figure 1. Pellicle formation in static liquid media. A. Top view of pellicles of $B$. subtilis IITKSM1 under different $\mathrm{NaCl}$ concentrations [(i) $W_{\mathrm{NaCl}}=0 \%$ (ii) $W_{\mathrm{NaCl}}=0.5 \%$ (iii) $W_{\mathrm{NaCl}}=1 \%$ (iv) $W_{\mathrm{NaCl}}=1.5 \%$ (v) $\left.W_{\mathrm{NaCl}}=2 \%\right]$ in liquid culture media after $c a .48 \mathrm{~h}$ of incubation at $30^{\circ} \mathrm{C}(24$ well plate, Well diameter- $15.5 \mathrm{~mm})$. All images are contrast enhanced for better visibility. B. Quantification of the wrinkles by plotting area covered $(A / A$ control $)$ and total number $\left(N_{\text {wrinkles }}\right)$ as a function of $W_{\mathrm{NaCl}}$. Data plotted is the mean obtained from the biological triplicates. C. Dry weight of pellicles ( $\left.W_{\text {pellicle}}\right)$ as a function of $W_{\mathrm{NaCl}}$. Data plotted is the mean obtained from three independent experiments. D. Secreted carbohydrate concentration $\left(C_{\text {carbohydrate }}\right)$ was estimated using phenolsulphuric acid method for varying $\mathrm{NaCl}$ concentrations. E. Optical density was measured for planktonic cells $\left(A_{600}\right)$ and crystal violet-stained surface adhered biofilm $\left(A_{540}\right)$ as a function of $W_{\mathrm{NaCl}}$. 
To shed light on this question, we measured the overall biomass of the pellicle $\left(W_{\text {pellicle }}\right)$ and the growth rate of the bacteria in the presence and absence of $\mathrm{NaCl}$. While there is an overall

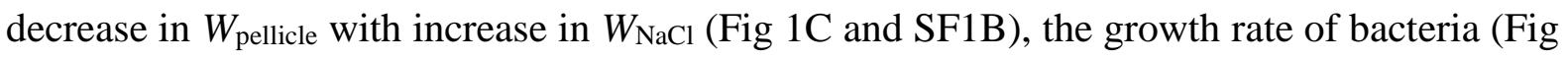
SF2), and the chemical nature of the biomolecules in the pellicle remained same as shown by FT-IR spectroscopy (Fig. SF3). Concomitant to the observed decrease in $W_{\text {pellicle, we observed }}$ a systematic decrease in the concentration of secreted carbohydrates $\left(C_{\text {carbohydrate }}\right)$ with increase in $W_{\mathrm{NaCl}}$. These results are further supported by $\mathrm{NaCl}$-induced reduction of the surface adhered biofilm and associated increase in planktonic growth of bacteria, as measured via the optical densities at $540 \mathrm{~nm}$ and $600 \mathrm{~nm}$, respectively (Fig. 1E). These results suggest the possible reasons underlying the $\mathrm{NaCl}$-induced deviations in the architecture of the biofilms. The overall reduction of $W_{\text {pellicle }}$ may result in reduced in-plane stresses (27) and the decrease in $C_{\text {carbohydrate }}$ suggest a possible decrease in the overall modulus of the pellicles. Assuming the buckling instability (27), the decrease in modulus of the substrate (monolayer) is expected to result in the decrease of the wavelength and, in turn, in the increased number of wrinkles. Clearly, these observations reveal that $\mathrm{NaCl}$ allows controlling the three-dimensional architecture of pellicles.

To improve our understanding of the importance of $\mathrm{NaCl}$, we performed experiments on biofilms that are grown on agar surface and the results are summarized in Fig 2. Clearly, we observed an increase in the rate of lateral expansion of B. subtilis biofilms in the presence of 2 wt. $\% \mathrm{NaCl}$ (Fig 2C), in contrast to the earlier reports showing a reduction in surface motility in the presence of $\mathrm{NaCl}(20,28-32)$. As we have shown in Fig SF4, only a limited concentrations of $\mathrm{NaCl}\left(W_{\mathrm{NaCl}}<2.5 \mathrm{wt} . \%\right)$ showed the increased surface motility of cells, while at higher concentrations there is a decrease in surface motility consistent with a previous study (20). To further support the enhanced surface motility in the presence of $\mathrm{NaCl}$, we assessed the ability of the biofilms to engulf foreign objects (33). For this purpose, we used PVDF membrane discs placed $1.5 \mathrm{~cm}$ apart along the lateral expansion direction. As expected, the 
biofilms in the presence of $\mathrm{NaCl}$ exhibited higher engulfing ability than the biofilms grown in the absence of $\mathrm{NaCl}$ (fig. 2D, 2E and fig. SF5). Evidently, the chosen low concentrations of $\mathrm{NaCl}$ allowed us to examine the increased motility of cells on the surface, revealing a novel characteristic of the cells in biofilms.

A

$\mathrm{B}$

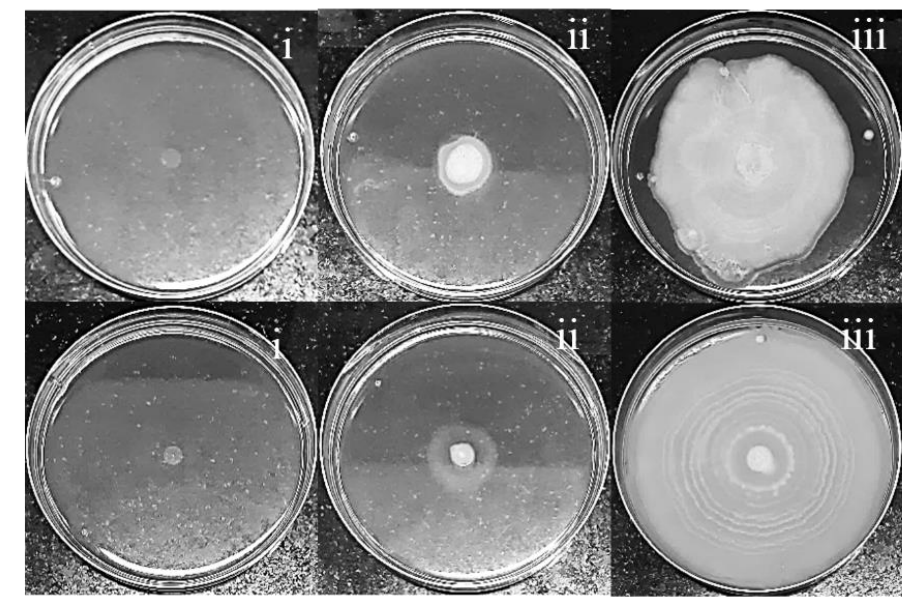

$\mathrm{C}$

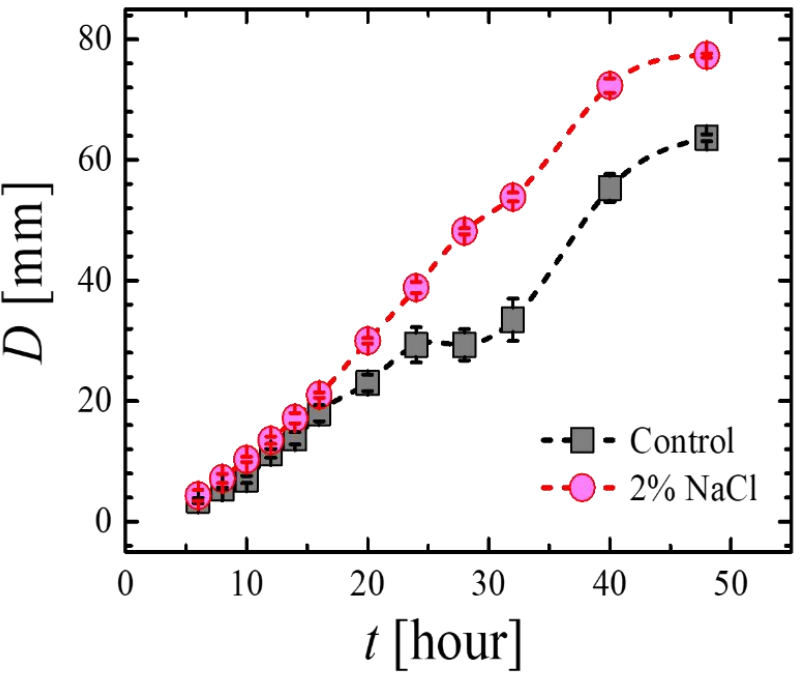

$\mathrm{D}$

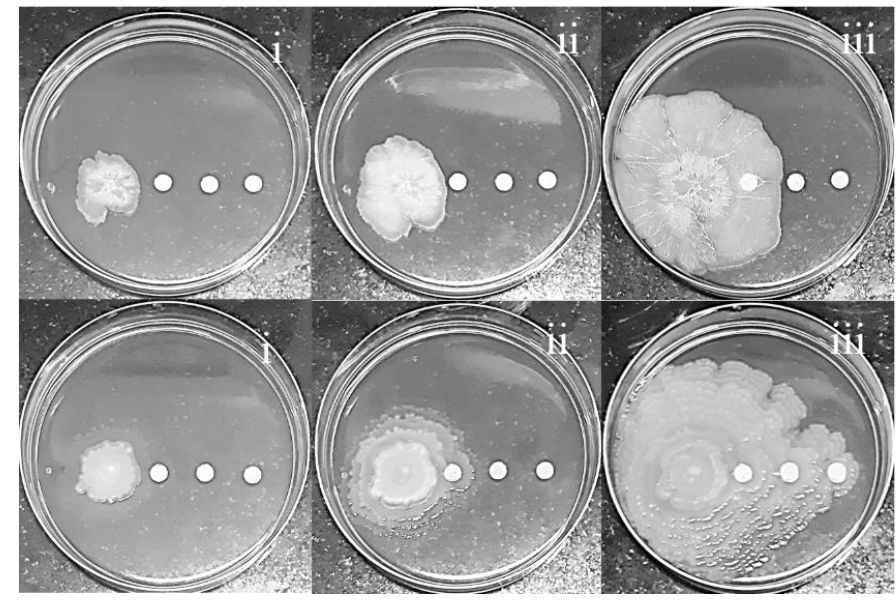

Figure 2. Biofilm expansion on agar surface. Representative time lapse [(i) $0 \mathrm{~h}$, (ii) $14 \mathrm{~h}$, (iii) $24 \mathrm{~h}$ ] images showing the lateral expansion (A) in absence of $\mathrm{NaCl}$, and (B) in presence of 2 wt.\% $\mathrm{NaCl}$. C. Diameter $(D)$ of the expanding biofilms $(\mathrm{n}=9)$ on $1.2 \%$ rich agar media Engulfment of PVDF membrane discs by $B$. subtilis IITKSM1 strain on $1.2 \%$ rich agar media (D) in the absence of $\mathrm{NaCl}$ and (E) presence of $2 \% \mathrm{NaCl}$. Diameter of the plates used in all the surface experiments is $90 \mathrm{~mm}$ ). 


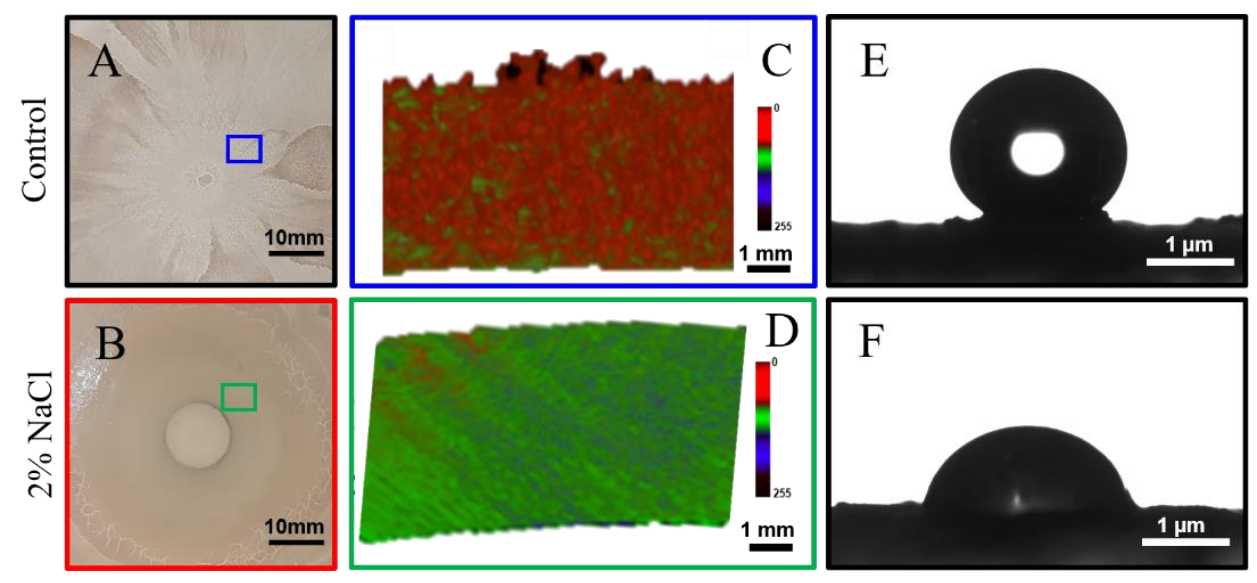

Figure 3. B. subtilis IITKSM1 colony morphology on agar with and without NaCl. Photograph focussing on the centre of a matured biofilm (A) in absence of $\mathrm{NaCl}$ and (B) presence of 2 wt.\% $\mathrm{NaCl}$. (C) and (D) X-ray Micro Tomographs corresponding the blue and green rectangles in A and B, respectively. Surface wettability of the colony centre grown $(\mathbf{E})$ without $\mathrm{NaCl}$ and $(\mathbf{F})$ with 2 wt.\% $\mathrm{NaCl}$ is captured via the contact angles of water on the respective surfaces.

In pellicle biofilms, we observed a significant reduction in the wrinkles on adding $\mathrm{NaCl}$. To verify if such variations exist in the topography of biofilms grown on the agar surface, we performed X-ray microtomography and the results are summarized in Fig 3. X-ray microtomography is a technique of choice as the matured biofilms are quite dense to be visualized by optical techniques. Assuming a chemical homogeneity at the lateral length scales of our microtomography experiments, the differences in the X-ray absorption could be related to the mean roughness of the surface. Corroborating the results of pellicle biofilms, the addition of $\mathrm{NaCl}$ showed a decrease in the surface roughness (Fig. 3C and 3D), suggesting the reduction in the formation of higher order structure of biofilms. Such reduction in higher-order structures is known to affect various properties of biofilms viz., penetration of foreign molecules into the biofilms (34), hydrophobicity of biofilms (35), and the mechanical tolerance of the biofilms (36-38). Here, we captured the wettability of the biofilm surface in the presence and absence of $\mathrm{NaCl}$ (Fig 3E and F). The contact angle of water at the center of the biofilms in the presence 
of $\mathrm{NaCl}$ was found to be $c a .77^{\circ}$, in contrast to $c a .140^{\circ}$ for biofilms formed without $\mathrm{NaCl}$. Thus, biofilms grown in the presence of $\mathrm{NaCl}$ showed a reduced hydrophobicity, which may facilitate the penetration of foreign molecules like drugs into the biofilms. These results demonstrate that $\mathrm{NaCl}$ controls the growth and formation of the B. subtilis biofilms. Does the presence of $\mathrm{NaCl}$ affects only at the macroscopic length scales of biofilm formation or also at the cellular and molecular scales?

\section{$\mathrm{NaCl}$ affects cell size and the kinetics of biofilm.}

To address this, we probed the spatiotemporal expansion by focusing on the bacterial cells at the expanding edge of the biofilm using time-lapse optical microscope. Interestingly, the length of the cells is $c a .25 \%$ smaller in the presence of $\mathrm{NaCl}$ (see SF6). We speculate that reduced cell size suggests cell division occurs at smaller sizes in the presence of $\mathrm{NaCl}$. As captured in Fig. 4A-B, expectedly, there is a clear increase in the velocity of the leading edge of the biofilms in the presence of $2 \mathrm{wt} \% \mathrm{NaCl}$. On a closer look at the micrographs (and the supporting videos 1 and 2), we observed different orientational behavior in the presence and absence of $\mathrm{NaCl}$. To quantify this, we measured the angle $\left(\theta_{i}\right)$ of the cells at the leading edge of the biofilm with respect to the expanding direction. Intriguingly, we observed a sharp peak at $\approx 10^{\circ}$ in the presence of $\mathrm{NaCl}$, in contrast to a much broader peak $\approx 70^{\circ}$ in the absence of $\mathrm{NaCl}$ (Fig. 4C). To understand how this shift in local alignment causes rapid edge expansion of biofilm, we followed the trajectory of all cells within the field of view. Fig. 4D captures the trajectories of chosen cells in the presence (red line) and absence (black line) of $\mathrm{NaCl}$. There is a directed motion of bacteria along the expanding direction in the presence of $\mathrm{NaCl}$. To further quantify the observed behavior, we deduced the mean square displacement of all cells within the field of view and shown in supporting information (Fig SF7). For clarity, in Fig. $4 \mathrm{~d}$, we show only the ensemble-averaged mean square displacements of cells along $(<$ $\left.\Delta x(t)^{2}>\right)$ and perpendicular $\left(<\Delta y(t)^{2}>\right)$ to the expanding direction, as a function of time. 
Mean square displacement in the absence of $\mathrm{NaCl}$ showed super-diffusive dynamics along both the directions: parallel as well as perpendicular to the expanding direction, i.e., $\left\langle\Delta x(t)^{2}>\approx\right.$ $<\Delta y(t)^{2}>\sim t^{\alpha}$ with $\alpha=1.35$ (Fig. 4D), consistent with the previous work (39). In contrast, we find diffusive dynamics of cells $(\alpha=1)$ in the presence of $\mathrm{NaCl}$. The microscopic reasons underlying the observed transition from super diffusive to diffusive dynamics is not yet clear and will be a focus of the upcoming work.
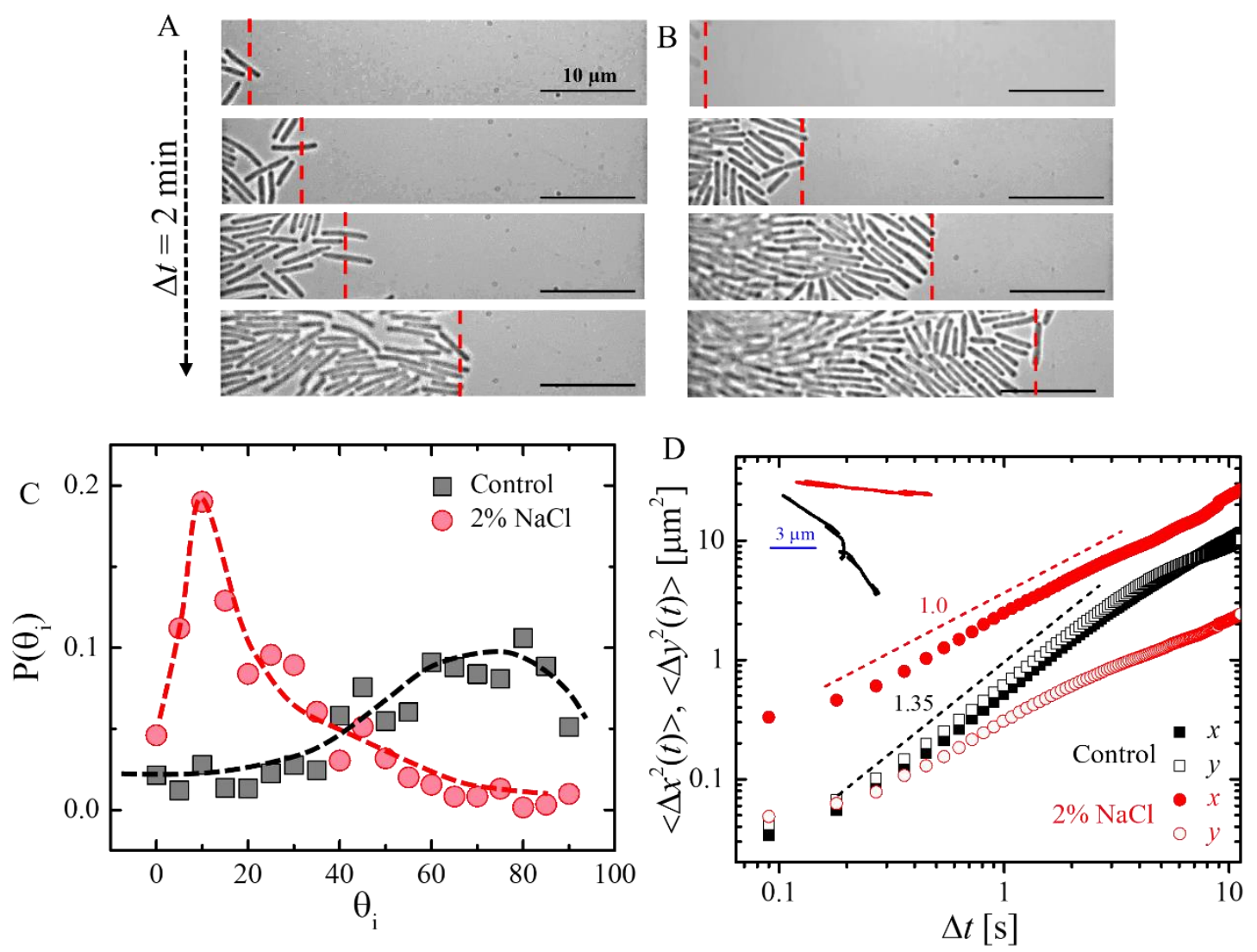

Figure 4. B. subtilis motility on agar with and without NaCl. Time series of optical micrographs after every 2-minute capturing the lateral expansion of biofilms, at the cellular level, in (A) the absence of $\mathrm{NaCl}$ and (B) in the presence of $2 \mathrm{wt} . \% \mathrm{NaCl}$. Microscopy experiments were performed $c a .13$ hours after the spotting of culture on the agar plates. C. The graph represents the probability of mean angle, obtained by averaging over different time frames, $P(\theta i)$ of the cells in the edge of expanding colony in presence (red circles) and absence (black squares) of $\mathrm{NaCl}$. D. Mean square displacement of motile cells in presence (red circles) and absence (black squares) of $\mathrm{NaCl}$, along with the representative trajectories (black and red corresponds to the trajectories in the absence and presence of $\mathrm{NaCl}$ ) of bacteria in both the cases. 
Interestingly, in the presence of $\mathrm{NaCl}$, we observed almost 7-8 times faster diffusion of cells along the expanding direction in comparison to the perpendicular direction. How do we understand this rapid expansion of bacteria (in the presence of $\mathrm{NaCl}$ ) parallel to the growth direction? As shown in Fig. 4C, the presence of $\mathrm{NaCl}$ induces an increased alignment of cells along the growth direction. In addition, we also observed (supporting videos 1 and 2) diffusion anisotropy, indicating the ease of cells to move along their length. Considering these observations, it is apparent that the bacteria would expand more readily along the expanding direction. The overall increase in the motility of the cells could also be supported by the reduced cell size (and the aspect ratio) in the presence of $\mathrm{NaCl}$. Thus, the presence of $\mathrm{NaCl}$ affects the cell size, and the kinetics of biofilm formation across length and time scales.

In the presence of $\mathrm{NaCl}$, it may be possible that the cells secrete different compounds that enables the expansion of biofilms, cellular motility, anisotropy in the diffusion along and perpendicular to the expanding direction, and transition from super diffusive to diffusive cellular motion. What are the molecular reasons through which $\mathrm{NaCl}$ affects the bacteria at the cellular level?

\section{Intracellular uptake of sodium and regulation of biofilm-to-motile transition}

To ascertain, whether the observed phenotypic changes were due to the increase in intracellular sodium concentration, we have used inductively coupled plasma mass spectrometry (ICP-MS). The increase in the total amount of sodium inside the cells after 12, 24 and 48 hours in the presence of 2 wt.\% $\mathrm{NaCl}$ (Fig. 5A), revealed ca. 6 times greater uptake of sodium. Therefore, it is highly likely that the increased intracellular levels of sodium upon $\mathrm{NaCl}$ treatment may be the factor governing the changes in biofilm formation of B. subtilis. To further examine whether the increased concentration of $\mathrm{NaCl}$ was responsible for the increased motility of the cells, we examined the expansion of biofilms in the presence of amiloride, a well-known 
inhibitor of sodium ion channel (40). As seen in Fig. 5B and 5C, $0.8 \mathrm{mM}$ amiloride inhibited the motility of the cells in the presence of $\mathrm{NaCl}$. Notably, both in the presence and absence of $\mathrm{NaCl}$, the biofilm expansion was similar when treated with amiloride, suggesting the specific $\mathrm{NaCl}$ uptake resulted in the observed phenotypes. How does the cellular uptake of $\mathrm{Na}^{+}$manifest into changes at the molecular level?

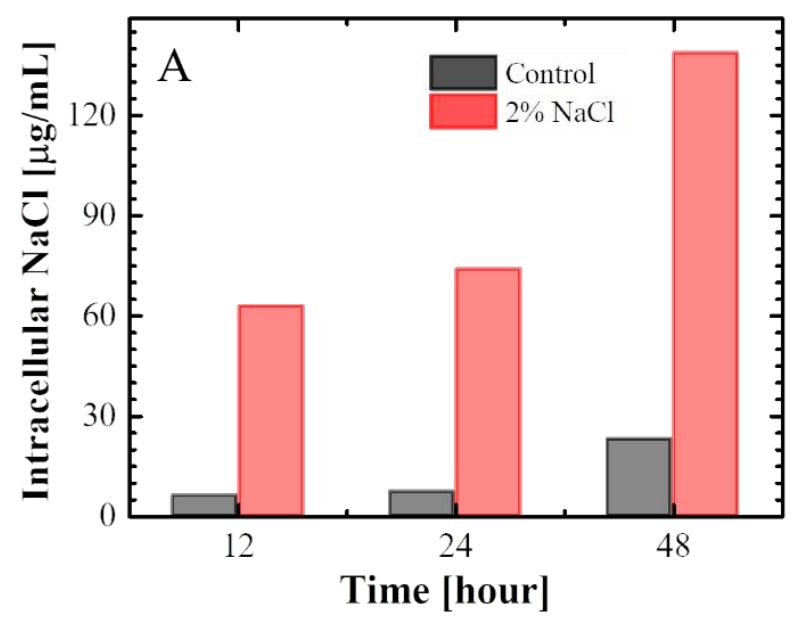

Figure 5. Total intracellular sodium uptake by B. subtilis IITKSM1 and

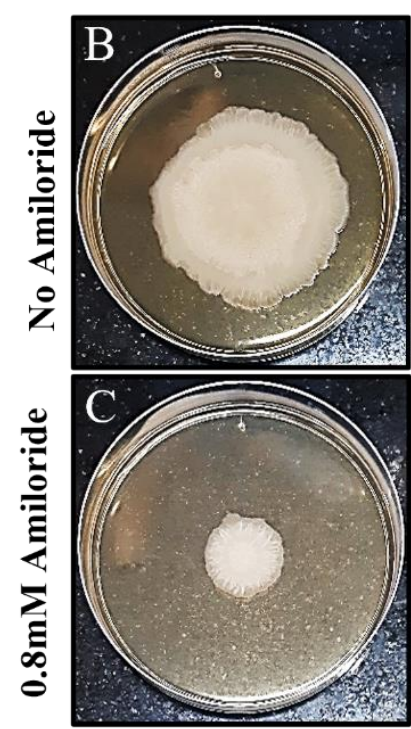

$0 \% \mathrm{NaCl}$

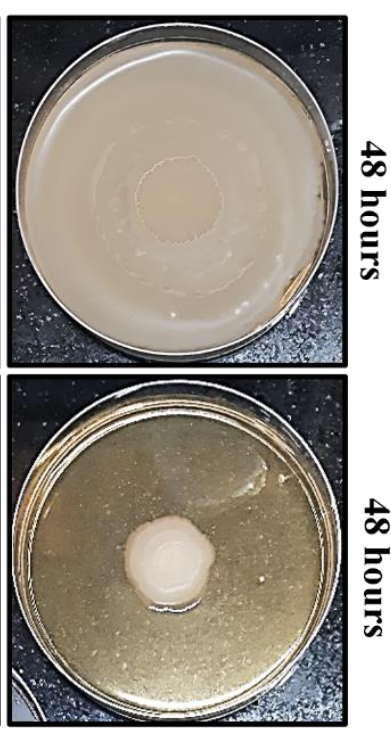

$2 \% \mathrm{NaCl}$
Effect of sodium channel blocker on

biofilm colony motility.

A. Intracellular sodium concentration in the absence and presence of 2 wt. $\% \mathrm{NaCl}$ as measured by ICP-MS. Motility of B. subtilis IITKSM1 on rich medium with (B) no $\mathrm{NaCl}$ and (C) 2 wt.\% $\mathrm{NaCl}$ in presence and absence of $0.8 \mathrm{mM}$ Amiloride, as observed after 48 hours.

To address this question, we quantified the relative change in gene expression upon the addition of $\mathrm{NaCl}$, using qRT-PCR to measure the expression level of selected genes relevant for the motility of cells and the formation of biofilms. As seen in (Fig. 6A), the expression of tapA, 
encoding an important component for biofilm formation is decreased by ca. 4-fold. Similarly, there is $c a .3$-fold decrease in the master regulator genes ( $\operatorname{slr} R$ and $s p o 0 A$ ), and $c a .2$-fold reduction in epsE, the matrix producing gene. Conceivably, the reduction in the expression of slrR, spoOA, tapA and epsE, in turn, impacts (diminishes) the formation of pellicles and biofilms. On the other hand, there is $c a$. 2-fold increase in the expression of swarming gene swrA and chemotaxis gene cheW in the presence of $\mathrm{NaCl}$, which likely contributes to the increased motility of the bacterial cells.

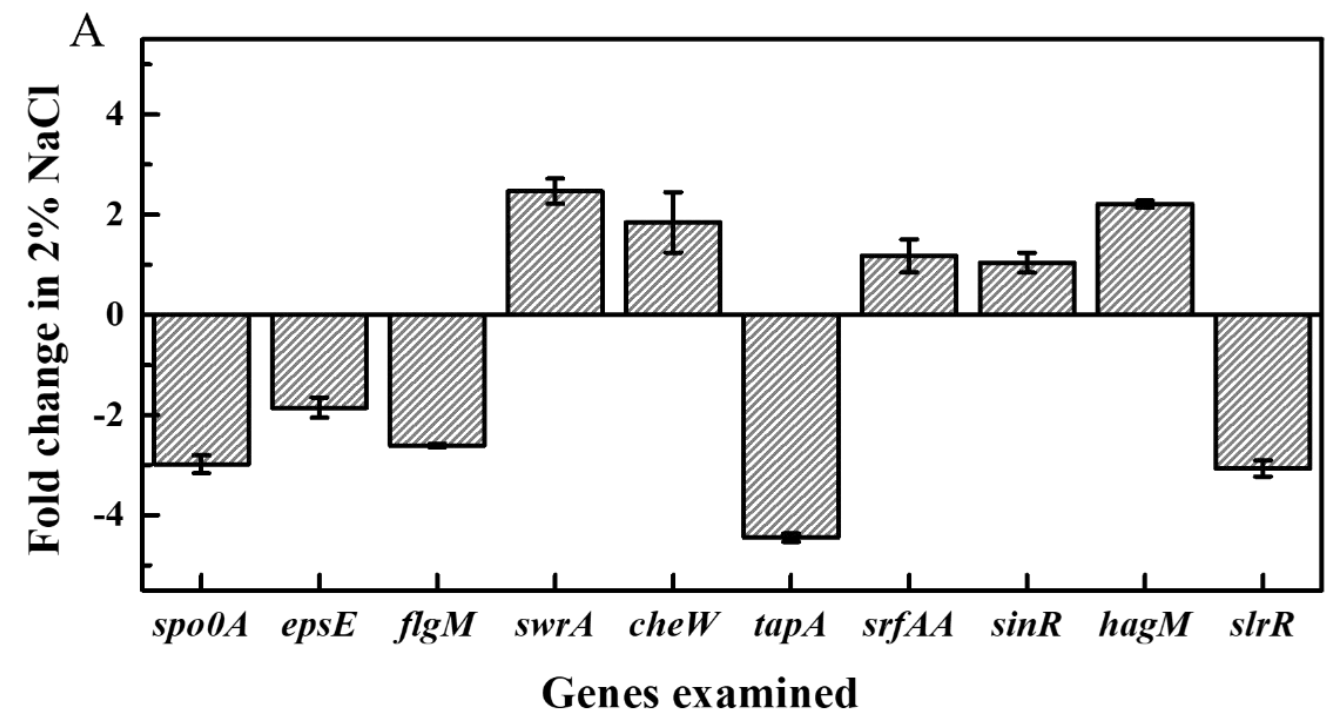

B

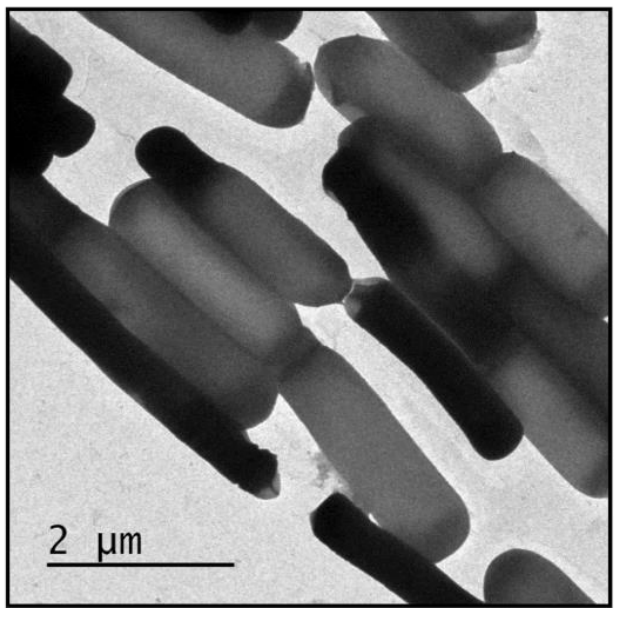

$\mathrm{C}$

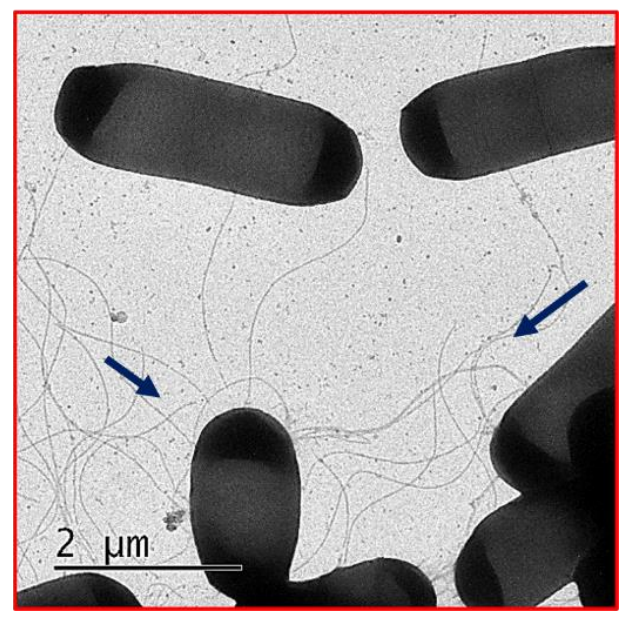

Figure 6. Mutually exclusive lifestyle between biofilm and planktonic state of $B$. subtilis IITKSM1 under the influence of $\mathbf{N a C l}$. A. NaCl-induced changes in the expression level selected genes relevant for motility and biofilm formation B. TEM images showing cells grown without $\mathrm{NaCl}$. C. Cells grown with 2 wt.\% $\mathrm{NaCl}$ in liquid 
media. The slender structures in (C), as pointed by the dark blue arrows, show the predominant presence of flagella in the presence of $2 \mathrm{wt} . \% \mathrm{NaCl}$.

Surprisingly, in the presence of $\mathrm{NaCl}$, there is ca. 2-fold increase in expression of hag gene, which is involved in the biosynthesis of flagella and there is a ca. 3-fold down-regulation of slrR gene that initiate the formation of biofilms by promoting cell chaining $(41,42)$. These changes in the gene expression, suggests the possibility of prominent differences at the level of individual cells upon $\mathrm{NaCl}$ treatment. To this end, we performed transmission electron microscope measurements of cells grown in the presence and absence of $\mathrm{NaCl}$. As seen in fig. $6 \mathrm{~B}$, in the absence of $\mathrm{NaCl}$, most of the cells were chained, while in the presence of $\mathrm{NaCl}$ (Fig 6C) the cells were less chained. These observations corroborate well with the SEM micrographs of matured biofilms, shown in Fig. SF8. Strikingly, we observed that in the presence of $\mathrm{NaCl}$, most of the cells were abundantly flagellated, in contrast to the nonflagellated cells in the absence of $\mathrm{NaCl}$. Thus, the enhanced motility of bacteria in the presence of $2 \mathrm{wt} . \% \mathrm{NaCl}$ could be due to the reduction in chaining and induction of flagella synthesis.

\section{Conclusions}

B. subtilis is a common soil-dwelling bacterium typically associated with plant roots, forming versatile biofilms with heterogeneous subpopulations. The subpopulations use a bet-hedging strategy to choose between either biofilm formation or flagella-mediated motility (43). In this study, we have characterized the importance of $\mathrm{NaCl}$ as a chemical cue and its potential influence the bistable switch that controls the biofilm (sessile) versus flagella-mediated motility decision. We found that a range of $\mathrm{NaCl}$ concentrations altered the biophysical properties, cellular morphology, gene expression and behavior of biofilms. The presence of $\mathrm{NaCl}$ caused 
a reduction in wrinkles, the dry weight and carbohydrate content of the pellicles, and reduced hydrophobicity, cumulatively indicating a major change in the biofilm architecture and properties (Figs 1-3). Salt enhanced the lateral expansion of cells and their ability to engulf foreign objects (Fig 2). These observations led to a key finding of the study, which is the increase in the percentage of a motile subpopulation within the bacterial biofilms. Cell motility was facilitated by the salt-induced increase in the flagellation of B. subtilis as shown by the transmission electron micrographs (Fig 6) and corroborated by the rapid anisotropic diffusion of cells (Fig 4). The salt-induced changes in the expression of several relevant genes strongly supported the decision of cells to adapt motility and limit biofilm formation. Upon exposure to $\mathrm{NaCl}$, the biofilm colony expresses major swarming, surfactin and flagellin genes, suggesting the possible reasons for the observed increase in the lateral expansion rates of the biofilms (Fig. 2 and 4). Simultaneously, in the presence of $\mathrm{NaCl}$, we observe a decreased expression in biofilm formation genes, such as tapA, slrR genes and negative flagellar regulator flgM. Thus our data is concordant with the proposed model of a strict and reverse regulation in the transcription of biofilm matrix genes during motility (44). In addition, the significant down regulation of epsE in the presence of $\mathrm{NaCl}$ might underlie the decrease in the carbohydrate concentration (Fig. 1D) and, in turn, the changes in the architecture of the pellicle biofilms

From the standpoint of biofilm, multicellular adaptation happens via surface motility of bacteria and hence, it would be noteworthy to define how the chemical cues like $\mathrm{NaCl}$ act as a switch for biofilm-to-motility transition. Previous studies have discussed the impact of stressors like antibiotics and the presence of other competitive species in their ability to induce short-range or long-range orientational order of cells and bacterial motility, underlying the necessity for bacterial species to move towards more favorable niches. (1). Additionally, motility is essential to engulf foreign colonies in a competitive environment in B. subtilis biofilms (34). $\mathrm{NaCl}$ may act as a chemical cue for $B$. subtilis cells to enhance motility, which 
may provide competitive benefits in nature. Importantly, how chemicals typically encountered by bacterial cells in their environment influences the multicellular systems is less understood (45). Various chemicals have distinct effects on the ability of bacterial species to form biofilms. For instance, $\mathrm{CaCl}_{2}$ is known to inhibit surface motility and increase biofilm formation (46), while Magnesium ions are showed to inhibit biofilm formation (47). We believe that $\mathrm{NaCl}$ could act as a chemical trigger to enhance motility and either to promote biofilm spread or to expand to new niches. Although, biofilms are sessile, within their extracellular matrix, a subpopulation of motile cells enables biofilm spreading (3). Furthermore, salt mediated changes in the cellular states within the biofilm may act as environmental cue for dispersal of subpopulations from mature biofilms. Chemical-mediated dispersal is an active mechanism of subpopulation escaping from mature or aging biofilms. For instance, dispersal in mature $B$. subtilis is triggered by D-amino acids (48). We demonstrated that the architectural and gene expression changes induced by salt are specifically mediated by cellular uptake of salt through sodium ion channels. Overall, we present evidence that salt can reprogram gene expression, alter cellular morphology and the state of cells to adapt to motility, which may facilitate the bacterial colony escape or expansion.

Besides, our study may highlight the possibility for augmenting the efficacy of antibiotic treatments. It is well known that biofilms are refractory to antibacterial agents owing to various reasons including the sessile state and reduced diffusion of the drugs in the biofilm matrix. For instance, biofilm formation is an essential feature of $B$. subtilis that can confer resistance to antimicrobial agents (49). Our findings on $\mathrm{NaCl}$-mediated biofilm-motility transition may additionally highlight an antibiotic evasion strategy. Since salt enhanced the motility of a subpopulation of cells, those motile and dividing cells can be targeted effectively by antibiotics than the sessile cells in the biofilm. To summarize, the dynamic nature of the subpopulations 
within bacterial biofilms and how their cellular states can be influenced purely by the chemical cues offer insights into the evolution of biofilms and their versatility in nature.

\section{Materials and Methods}

\section{Bacterial strains and Media:}

The bacterial culture used in the study is Bacillus subtilis IITKSM1. The strain isolation and sequencing information are shown in our earlier work (26). The B. subtilis IITKSM1 was grown on rich medium containing Yeast Extract (1\%), peptone (2\%) and dextrose (2\%). In addition to the rich medium, different $\mathrm{NaCl}$ concentrations ranging from 0 to $2 \mathrm{wt} \%$, amounting to a maximum molarity of $c a .0 .37 \mathrm{M}$, were used. The pellicle formation and colony architecture assays were performed in rich media and in varied concentrations of $\mathrm{NaCl}$ described above. Complementary experiments were performed using minimal media glutamate glycerol (MSgg) broth as used in (50) $(95.37 \mathrm{ml}$ sterile MQ water, $7.5 \mathrm{ml} 0.1 \mathrm{M}$ phosphate buffer, $30 \mathrm{ml} 0.5 \mathrm{M}$ MOPS pH 7, $3 \mathrm{ml} 0.1 \mathrm{M} \mathrm{MgCl}_{2}, 1.05 \mathrm{ml} 0.1 \mathrm{M} \mathrm{CaCl}_{2}, 0.75 \mathrm{ml} 0.01 \mathrm{M}$ $\mathrm{MnCl}_{2}, 0.9 \mathrm{ml} 8.35 \mathrm{mM} \mathrm{FeCl}_{3}, 0.15 \mathrm{ml} 1.0 \mathrm{mM} \mathrm{ZnCl}, 0.03 \mathrm{ml} 0.01 \mathrm{M}$ thiamine $\mathrm{HCl}, 1.5 \mathrm{ml}$

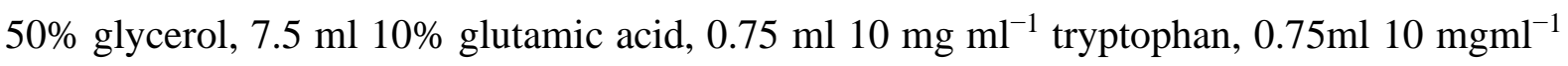
phenylalanine and $0.75 \mathrm{ml} 10 \mathrm{mg} \mathrm{ml}^{-1}$ threonine $150 \mathrm{ml}^{-1}$ media) or on MSgg plates supplemented with $1.5 \%$ agar and with appropriate concentrations of $\mathrm{NaCl}$. All medium components were made as solutions in sterile MQ water and sterilized by either autoclaving or filter sterilization and mixed aseptically before use.). The culture was maintained on rich and LB Agar plates. The primers used in the study are listed in Table 1.

\section{Pellicle formation assay}

For floating pellicle assays, B. subtilis strain IITKSM1 was cultured in rich medium and minimal medium in Tarsons ( 24 well) plates at $30^{\circ} \mathrm{C}$ for 48 hours and 72 hours, respectively. 
The $\mathrm{NaCl}$ concentrations were varied in the in each well (51). The number of pellicles, pellicle fold width and area of folds in the pellicle was analyzed using ImageJ software (52) and plotted using Origin Pro 9.1 software (OriginLab corporation [http://www.OriginLab.com]). For quantifying the dry weight of the formed pellicle, $1 \mathrm{ml}$ of $100 \%$ ethanol was carefully poured under the pellicle using a pipette to lift it from the surface of the liquid. The obtained pellicles were vacuum dried, and the weights were measured. The data shown in Fig. 1 are representative of three independent experiments.

\section{Surface motility Assay}

Bacteria were grown in rich medium at $30{ }^{\circ} \mathrm{C}$ for 12 hours at $200 \mathrm{rpm}$. The inoculum was adjusted to obtain $\mathrm{OD}_{600}$ 1.0. Three microliters of cell suspension were spotted on $1.2 \%$ agar rich medium without and with $2 \mathrm{wt} . \% \mathrm{NaCl}$ and grown at $30{ }^{\circ} \mathrm{C}$ for up to 48 hours. The time lapse photos were captured using straight forward mobile camera. To confirm the effect of $\mathrm{NaCl}$ is due to the sodium ions, a sodium channel blocker named amiloride at $0.8 \mathrm{mM}$ concentration in the agar medium. To observe the colony morphology in minimal MSgg media, similar procedure was followed with varying $\mathrm{NaCl}$ concentrations.

\section{Disc Engulfment assay}

The extent of engulfment by $B$. subtilis IITKSM1 in presence and absence of $\mathrm{NaCl}$ was performed using methods discussed in reference (34). Three PVDF membrane discs of $6 \mathrm{~mm}$ diameter were placed at $1.5 \mathrm{~cm}, 3 \mathrm{~cm}$, and $4.5 \mathrm{~cm}$ from the inoculated biofilm colony. For each B. subtilis IITKSM1 colony, the number of PVDF discs engulfed by the expanding biofilm colony were captured and quantified. The results presented here are the measurements of at least 4 different colonies at each time point. 


\section{Optical Microscopy and imaging of Motility}

B. subtilis IITKSM1 cells swarming on surface of rich agar medium were imaged with timelapse optical microscope (Olympus BX40 microscope) using 100X magnification (Olympus, Japan). Optical micrographs of the expanding edges, in the presence and absence of $\mathrm{NaCl}$, were obtained $c a .14$ hours after spotting on the agar plate.

\section{Image Analysis and Bacteria Tracking.}

Image analysis and cell-tracking were performed using the open access package CellProfiler [CellProfiler Project [http://www.cellprofiler.org]]. The input images were first converted to gray scale images, whose contrast is enhanced by implementing inbuilt module named "EnhanceOrSuppressFeatures" in the cellProfiler. Using the contrast enhanced image, we obtained the binary image by implementing "otsu-thresholding method". Thresholded images as obtained are classified into cells and background using watershed algorithm. Cell orientation and its dimension were calculated by using "objectSizeShape" module in CellProfiler. Such images were subsequently used for tracking the trajectory of individual cell using a standard particle-tracking algorithm based on a "Follow neighbor criterion" in successive frames. Further, the trajectories were analyzed with python scripts to obtain mean square displacements.

\section{X-ray Microtomography}

X-ray microtomography analysis was done using Bruker Skyscan 1275 micro-CT. A small sample $(9 \mathrm{~mm} \times 5 \mathrm{~mm})$ was excised from the proximity of the centre of the mature biofilm on agar. The sample was placed on a rotation stage and irradiated with X-rays on the surface of the biofilm by rotating it $180^{\circ}$ in equally spaced increments. At every angle three projections 
were obtained and averaged to obtain a 2D projection. The magnification chosen for the samples within the field of view which corresponded to voxel resolution of $\sim 5 \mu \mathrm{m}$ (53).

\section{Analysis of Surface Wettability}

The $B$. subtilis IITKSM1 cells were grown for 48 hours on rich agar medium. The contact angle measurement was determined using a goniometer (KRUSS-Drop Shape Analyzer-DSA 25E). The water droplet was placed close to the proximity of mature biofilm centre and the contact angles were measured for different samples. The drop profile was processed using the image analysis package ADVANCE software, KRUSS, GmbH, Germany.

\section{Transmission Electron Microscopy (TEM)}

For investigation of flagella by TEM (54), B. subtilis IITKSM1 was grown for 12 hours in rich medium without and with $2 \% \mathrm{NaCl}$ supplementation. The cells grown on rich medium broth for ca. 14 hours were absorbed onto copper grids. The grids were washed twice with PBS. Negative staining of the cells was done using $1 \%$ freshly prepared uranyl acetate. Samples were viewed in FEI Technai G2 20 twin TEM.

\section{RNA isolation and Q-PCR}

RNA was isolated from 12 -hour culture of $B$. subtilis IITKSM1 grown at $37^{\circ} \mathrm{C}$. The procedure was followed from (55). Briefly, 10ml of 12-hour culture was centrifuged and pelleted. The pellet was frozen and stored at $-80^{\circ} \mathrm{C}$ for 24 hours. The pellet was later dissolved in $0.5 \mathrm{ml}$ of lysis buffer (30mM, 10mM EDTA with $10 \mathrm{mg} / \mathrm{ml}$ lysozyme) and incubated at $37^{\circ} \mathrm{C}$ for 30 minutes. This was followed by addition of $1 \mathrm{ml}$ of Trizol reagent and $0.3 \mathrm{ml}$ of chloroform to the tube. The tube was centrifuged at $15000 \mathrm{rpm}$ for 20 minutes at $4^{\circ} \mathrm{C}$. The top aqueous layer was taken, and equal volume of isopropanol was added to it and stored at $-20^{\circ} \mathrm{C}$ for 2 to 4 hours. The precipitated RNA was centrifuged at $15000 \mathrm{rpm}$ for 20 minutes at $4^{\circ} \mathrm{C}$. The obtained 
pellet was washed with $0.5 \mathrm{ml}$ with ice cold $70 \%$ ethanol. Pellets were dissolved in $20 \mu \mathrm{l}$ double distilled water. The isolated RNA $(2 \mu \mathrm{g})$ was converted to cDNA with Quantitect Reverse Transcription kit (Qiagen, USA). The genes chosen were epsE, tapA, spoOA, swrA, srfAA, flgM and cheW with rpsE as housekeeping gene. Real time PCR was performed using Promega GoTaq Green Q-PCR Master mix, one step with the following conditions for activation of enzyme at $95^{\circ} \mathrm{C}$ for 30 s, followed by 40 cycles of denaturation at $95^{\circ} \mathrm{C}$ for $30 \mathrm{~s}$, annealing at $55^{\circ} \mathrm{C}$ for 1 minute. The melt curve analysis was performed at $65^{\circ} \mathrm{C}$ for 1 minute in $\mathrm{CFX}$ connects TM Real-time PCR detection system (Bio-Rad, USA). The primers used are listed in Table 1. Relative mRNA levels were determined by fold change. This was calculated by 2$\Delta \Delta \mathrm{CT}$ using the mRNA level of rpsE which represents the house keeping gene for comparison (56).

\section{Quantification of planktonic cells, surface adhered Biofilm formation and amount of} secreted carbohydrates.

To determine how the planktonic cells and surface adhered biofilms alter under different $\mathrm{NaCl}$ concentrations, the $B$. subtilis IITKSM1 was grown in Tarsons (96 well) plates. To form biofilms, the cells were grown for up to 48 hours in the standardized rich medium at $30^{\circ} \mathrm{C}$ as static culture (57). The secreted carbohydrate concentrations were measured from the culture filtrate. The concentrations were determined with phenol sulfuric acid method using glucose as a standard (58). The data represented here are from 3 independent experiments.

\section{Fourier transform infrared spectroscopy (FTIR)}

A mature pellicle of $B$. subtilis IITKSM1, grown for $c a$. two days on the rich medium (with and without $\mathrm{NaCl}$ ) was cautiously separated. The pellicle was dried and lyophilized. Infrared spectroscopic measurements of the samples were performed on Bruker Tensor 27 IR 
spectrophotometer (Bruker Corporation, USA, KBr Beam splitter). All spectral readings were smoothened using the standard automatic smooth function (59).

\section{Estimation of intracellular concentration of sodium in B. subtilis IITKSM1 cells}

Bacterial cells with balanced growth were obtained from $0 \%$ and $2 \% \mathrm{NaCl}$ supplemented medium were pelleted, and the medium was removed. The obtained pellets were washed using $1 \mathrm{M}$ Tris- $\mathrm{Cl}$ to remove excess ions and medium without lysing the cells. The cells were lysed in $2 \mathrm{ml} 1 \%$ HNO3 followed by sonication to break up the lysate. ICP-MS (Agilent 7900, USA) was used to estimate the intracellular concentration of potassium and sodium within the lysates. The concentrations measured were then used to estimate the average number of moles of sodium ions in a single cell, which can further be used to determine the intracellular concentration of the sodium (60). 
Table 1

\begin{tabular}{|c|c|}
\hline TapAFW & 5'AACCGACAGTCCCTAAAAAAG3' \\
\hline TapARE & 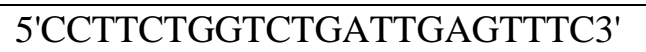 \\
\hline Spo0AFW & 5'TTCAACACAACCGCAAGCC3 \\
\hline Spo0ARE & 5'TTTCCTCTGCTCCATGCCAC3' \\
\hline RpsEFW & 5'ACTGGAGTTATCGCTGGAGG3' \\
\hline RpsERE & 5'AGTGTTGCACGAATCATGTTG3' \\
\hline SrfAAFW & 5'CGTATAGAGAGAGAAGCGGAG3' \\
\hline SrfAARE & 5'AAGTGGTGAATGUCAAGAAA3' \\
\hline SwrAFW & $\begin{array}{l}\text { 5'TCAGCTACAAAAGCACTAAGTC3' } \\
\end{array}$ \\
\hline SwrARE & 5'ATGTTTGGCGATTCCTC3' \\
\hline CheWFW & 5'CTTCATCCACAATCCAGCC3' \\
\hline CheWRE & 5'GGGGTAATCAATTTACGCGG3' \\
\hline EpsEFW & 5'TATGATGAGAGCGAGTGCC3' \\
\hline EpsERE & 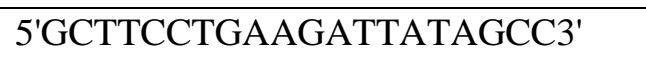 \\
\hline FlgMFW & 5'AAAAACTGCTGCACAGCC3' \\
\hline FlgMRE & 5'GCGTCTACTTTGTATGACCC3' \\
\hline HagMFW & 5'TCAAACCAACGTGCTAAACTTG3' \\
\hline HagMRE & 5'AAATCGCTCATTTCTTTCGCC3' \\
\hline
\end{tabular}




\section{Acknowledgments}

We thank Irfan Qayoom and Ashok Kumar for allowing access to X-ray Micro CT facility, Krishnacharya Khare for contact angle measurements, Akshat Verma and Abhas singh for ICPMS. We acknowledge the valuable suggestions from Muthukumaran Venkatachalapathy and Dharmaraja Allimuthu. We thank the funding from IITK. SM thank INSA and IYBA for the funding and fellowship, respectively.

\section{References}

1. Grobas I, Bazzoli DG, \& Asally M (2020) Biofilm and swarming emergent behaviours controlled through the aid of biophysical understanding and tools. Biochem. Soc. Trans. 48(6):2903-2913.

2. Mazza MG (2016) The physics of biofilms-An introduction. J. Phys. D: Appl. Phys. 49(20):203001.

3. Be'er A \& Ariel G (2019) A statistical physics view of swarming bacteria. Mov. Ecol. $7(1): 9$.

4. Daniels R, Vanderleyden J, \& Michiels J (2004) Quorum sensing and swarming migration in bacteria. FEMS Microbiol. Rev. 28(3):261-289.

5. Xavier JB (2011) Social interaction in synthetic and natural microbial communities. Mol. Syst. Biol. 7(1):483.

6. Benarroch JM \& Asally M (2020) The microbiologist's guide to membrane potential dynamics. Trends Microbiol 28(4):304-314.

7. Bhattacharyya S, Walker DM, \& Harshey RM (2020) Dead cells release a 'necrosignal' that activates antibiotic survival pathways in bacterial swarms. Nat. Commun. 11(1):4157. 
8. Butler MT, Wang Q, \& Harshey RM (2010) Cell density and mobility protect swarming bacteria against antibiotics. Proc. Natl. Acad. Sci. 107(8):3776-3781.

9. Flemming H-C \& Wingender J (2010) The biofilm matrix. Nat. Rev. Microbiol. 8(9):623-633.

10. Harshey RM (2003) Bacterial Motility on a Surface: Many Ways to a Common Goal. Annu. Rev. Microbiol. 57(1):249-273.

11. Henrichsen J (1972) Bacterial surface translocation: a survey and a classification. Bacteriol. Rev. 36(4):478-503.

12. Hoffman LR, et al. (2005) Aminoglycoside antibiotics induce bacterial biofilm formation. Nature 436(7054):1171-1175.

13. Jones RM, et al. (2015) Lactobacilli Modulate Epithelial Cytoprotection through the Nrf2 Pathway. Cell Rep. 12(8):1217-1225.

14. Kearns DB (2010) A field guide to bacterial swarming motility. Nat. Rev. Microbiol. 8(9):634-644.

15. Liu Y, et al. (2015) Genomic insights into the taxonomic status of the Bacillus cereus group. Sci. Rep. 5(1):14082.

16. Liu Z, et al. (2018) A Genome-Wide Screen Identifies Genes in RhizosphereAssociated $<$ em $>$ Pseudomonas $</$ em $>$ Required to Evade Plant Defenses. $m$ Bio 9(6):e00433-00418.

17. Mah T-F (2012) Biofilm-specific antibiotic resistance. Future Microbiol. 7(9):10611072.

18. Meredith HR, Lopatkin AJ, Anderson DJ, \& You L (2015) Bacterial Temporal Dynamics Enable Optimal Design of Antibiotic Treatment. PLoS Comput. Biol. 11(4):e1004201. 
19. Prindle A, et al. (2015) Ion channels enable electrical communication in bacterial communities. Nature 527(7576):59-63.

20. Rath H, et al. (2020) Impact of high salinity and the compatible solute glycine betaine on gene expression of Bacillus subtilis. Environ. Microbiol. 22(8):3266-3286.

21. Sharma D, Misba L, \& Khan AU (2019) Antibiotics versus biofilm: an emerging battleground in microbial communities. Antimicrob. Resist. Infect. Control. 8(1):1-10.

22. Vlamakis H, Chai Y, Beauregard P, Losick R, \& Kolter R (2013) Sticking together: building a biofilm the Bacillus subtilis way. Nat Rev Microbiol. 11(3):157-168.

23. Wang Y, et al. (2011) Phenazine-1-carboxylic acid promotes bacterial biofilm development via ferrous iron acquisition. J. Bacteriol. 193(14):3606-3617.

24. Karatan E \& Watnick P (2009) Signals, regulatory networks, and materials that build and break bacterial biofilms. Microbiol. Mol. Biol. Rev. 73(2):310-347.

25. Grobas I, Polin M, \& Asally M (2021) Swarming bacteria undergo localized dynamic phase transition to form stress-induced biofilms. Elife 10:e62632.

26. Anand MP, Hariharan VC, Shankar H, Singh A, \& Saravanan M (2019) Genome Sequence of Bacillus subtilis subsp. subtilis strain IITK SM1, Isolated from Kitchen Waste Compost. Microbiol. Resour. Announc. 8(6).

27. Trejo M, et al. (2013) Elasticity and wrinkled morphology of Bacillus subtilis pellicles. Proc Natl Acad Sci 110(6):2011-2016.

28. De Silva PM \& Kumar A (2018) Effect of sodium chloride on surface-associated motility of Acinetobacter baumannii and the role of AdeRS two-component system. $J$ Membr. Biol. 251(1):5-13.

29. Soman V, Kumari S, Nath S, \& Elangovan R (2020) The Response of Bacterial Flagellar Motor to Stepwise Increase in $\mathrm{NaCl}$ Concentration. bioRxiv:2020.2008.2019.257477. 
30. Dewangan NK \& Conrad JC (2020) Bacterial motility enhances adhesion to oil droplets. Soft Matter 16(35):8237-8244.

31. Yang A, Tang WS, Si T, \& Tang JXJBJ (2017) Influence of physical effects on the swarming motility of Pseudomonas aeruginosa. Biophys. J. 112(7):1462-1471.

32. Havasi V, et al. (2008) Inhibitory effects of hypertonic saline on P. aeruginosa motility. J. Cyst. Fibros. 7(4):267-269.

33. Rosenberg G, et al. (2016) Not so simple, not so subtle: the interspecies competition between Bacillus simplex and Bacillus subtilis and its impact on the evolution of biofilms. NPJ Biofilms Microbiomes 2(1):1-11.

34. Steinberg N, et al. (2020) The extracellular matrix protein TasA is a developmental cue that maintains a motile subpopulation within Bacillus subtilis biofilms. Science Signalling 13(632):eaaw8905.

35. Werb M, et al. (2017) Surface topology affects wetting behavior of Bacillus subtilis biofilms. NPJ Biofilms Microbiomes 3(1):1-10.

36. Yan J, et al. (2019) Mechanical instability and interfacial energy drive biofilm morphogenesis. Elife 8:e43920.

37. Beroz F, et al. (2018) Verticalization of bacterial biofilms. Nat. Phys. 14(9):954-960.

38. Asally M, et al. (2012) Localized cell death focuses mechanical forces during 3D patterning in a biofilm. Proc Natl Acad Sci 109(46):18891-18896.

39. Ariel G, et al. (2015) Swarming bacteria migrate by Lévy Walk. Nat. Commun. 6(1):1-6.

40. Sugiyama S, Cragoe Jr EJ, \& Imae Y (1988) Amiloride, a specific inhibitor for the Na+-driven flagellar motors of alkalophilic Bacillus. J. Biol. Chem. 263(17):82158219. 
41. Cozy LM, et al. (2012) SlrA/SinR/SlrR inhibits motility gene expression upstream of a hypersensitive and hysteretic switch at the level of $\sigma(\mathrm{D})$ in Bacillus subtilis. Mol. Microbiol. 83(6):1210-1228.

42. Kobayashi K (2007) Bacillus subtilis Pellicle Formation Proceeds through Genetically Defined Morphological Changes. J. Bacteriol. 189(13):4920-4931.

43. Ryan-Payseur BK \& Freitag NE (2018) Bacillus subtilis Biofilms: a Matter of Individual Choice. mBio 9(6):e02339-02318.

44. Vlamakis H, Aguilar C, Losick R, \& Kolter R (2008) Control of cell fate by the formation of an architecturally complex bacterial community. Genes Dev 22(7):945953.

45. Smith V, et al. (2020) MogR Is a Ubiquitous Transcriptional Repressor Affecting Motility, Biofilm Formation and Virulence in Bacillus thuringiensis. Front. Microbiol. 11:610650-610650.

46. Mhatre E, et al. (2017) Presence of Calcium Lowers the Expansion of Bacillus subtilis Colony Biofilms. Microorganisms 5(1):7.

47. Oknin H, Steinberg D, \& Shemesh M (2015) Magnesium ions mitigate biofilm formation of Bacillus species via downregulation of matrix genes expression. Front Microbiol. 6(907).

48. Leiman SA, et al. (2013) D-amino acids indirectly inhibit biofilm formation in Bacillus subtilis by interfering with protein synthesis. J. Bacteriol. 195(23):53915395.

49. Sarikhani M, Kermanshahi RK, Ghadam P, \& Gharavi S (2018) The role of probiotic Lactobacillus acidophilus ATCC 4356 bacteriocin on effect of HBsu on planktonic cells and biofilm formation of Bacillus subtilis. Int. J. Biol. Macromol. 115:762-766. 
50. Newton R, Amstutz J, \& Patrick JE (2020) Biofilm formation by Bacillus subtilis is altered in the presence of pesticides. Access Microbiol 2(12).

51. López D, Fischbach MA, Chu F, Losick R, \& Kolter R (2009) Structurally diverse natural products that cause potassium leakage trigger multicellularity in \&lt;em\&gt;Bacillus subtilis\&lt;/em\&gt. Proc. Natl. Acad. Sci. 106(1):280.

52. Schneider CA, Rasband WS, \& Eliceiri KW (2012) NIH Image to ImageJ: 25 years of image analysis. Nat. Methods. 9(7):671-675.

53. Tisato N, et al. (2015) Microbial mediation of complex subterranean mineral structures. Sci Rep 5(1):15525.

54. Kirov SM, et al. (2002) Lateral flagella and swarming motility in Aeromonas species. J. Bacteriol. 184(2):547-555.

55. Villa-Rodríguez E, Ibarra-Gámez C, \& de los Santos-Villalobos S (2018) Extraction of high-quality RNA from Bacillus subtilis with a lysozyme pre-treatment followed by the Trizol method. J Microbiol Methods 147:14-16.

56. Pumirat P, et al. (2017) Effects of sodium chloride on heat resistance, oxidative susceptibility, motility, biofilm and plaque formation of Burkholderia pseudomallei. Microbiologyopen 6(4):e00493.

57. Nait Chabane Y, et al. (2014) Characterisation of pellicles formed by Acinetobacter baumannii at the air-liquid interface. PLoS One 9(10):e111660.

58. DuBois M, Gilles KA, Hamilton JK, Rebers PA, \& Smith F (1956) Colorimetric Method for Determination of Sugars and Related Substances. Anal. Chem 28(3):350356.

59. Baker MJ, et al. (2014) Using Fourier transform IR spectroscopy to analyze biological materials. Nat. Protoc. 9(8):1771-1791. 
bioRxiv preprint doi: https://doi.org/10.1101/2022.02.15.480532; this version posted February 15, 2022. The copyright holder for this preprint (which was not certified by peer review) is the author/funder. All rights reserved. No reuse allowed without permission.

60. Jensen MW, et al. (2015) Potassium stress growth characteristics and energetics in the haloarchaeon Haloarcula marismortui. Extremophiles 19(2):315-325. 